\title{
Simplest phonons and pseudo-phonons in field theory
}

\author{
Daniele Musso $^{1,2, \mathrm{a}_{\mathbb{C}}}$ \\ ${ }^{1}$ Universidad de Santiago de Compostela (USC), Santiago de Compostela, Spain \\ ${ }^{2}$ Instituto Galego de Física de Altas Enerxías (IGFAE), Santiago de Compostela, Spain
}

Received: 23 February 2019 / Accepted: 19 November 2019 / Published online: 4 December 2019

(c) The Author(s) 2019

\begin{abstract}
We study the emergence of Nambu-Goldstone modes due to broken translation symmetry in field theory. Purely spontaneous breaking yields a massless phonon which develops a mass upon introducing a perturbative explicit breaking. The pseudo-phonon mass agrees with Gell MannOakes-Renner relations. We analyze the simplest possible theories featuring gradient Mexican hats and describing space-dependent order parameters. We comment on homogeneous translation breaking and the connections with holographic Q-lattices.
\end{abstract}

\section{Introduction and motivation}

The breaking of spatial translations is fundamental to many physical systems, especially but not only in condensed matter. The lack of momentum conservation and the emergence of phonons are two important consequences of different kinds of translation symmetry breaking, explicit and spontaneous respectively. Typically the realization of translation symmetry breaking is technically complicated due to the spatial dependence of the fields. Although not being a conceptual obstruction, such complication usually requires non-analytic tools.

An important exception to this state of affairs has been explored in recent years within the holographic context, with the phenomenological aim of modeling strongly-correlated systems that break translations and lack a standard quasiparticle description. Holographic models geometrize the renormalization group flow of a conjectured quantum field theory by means of dual gravitational fields, often having a radial dependence in an otherwise symmetric and homogeneous space. Spatial features can be added, yet the computations get much more difficult since the equations of motion become partial differential equations.

\footnotetext{
a e-mail: daniele.musso@usc.es
}

Wide families of holographic models avoid the leap in complexity at the price of introducing conceptual subtleties. Such models are characterized by a homogeneous breaking of translation symmetry, where an internal global symmetry renders all the spacetime points of the broken phase equivalent [1-3]. ${ }^{1}$ Basic concepts like those of unit cell or commensurability [11] are thereby absent.

The present paper exports the idea of homogeneous translation symmetry breaking to standard, non-holographic field theory. Specifically, it studies the "Mexican hat potential" for translation symmetry breaking, both in a literal and a loose sense: literally, the mechanism of the Mexican hat is realized in the gradient sector; loosely, the aim at stake is to analyze simple prototypical field theory examples of translation symmetry breaking.

Phonons have been long considered in field theory, yet they are introduced ad hoc. This is the case for Fröhlich Hamiltonians describing electron-phonon interactions, and for effective field theories of elastic media [15]. Many phenomenological questions indeed are insensitive to the microscopic details. Nevertheless, there are reasons why elucidating the origin of phonons is important, both conceptually and phenomenologically: ${ }^{2}$

- Low-energy effective field theories relies on information about the symmetries: the number of low-energy degrees of freedom is in general expected to be given by counting theorems descending from the symmetry breaking pattern [16].

\footnotetext{
${ }^{1}$ Models featuring massive gravity [4-6] have been considered too, they fall into the same category because they can be realized through a Stueckelberg mechanism in terms of fields that enjoy a global symmetry $[7,8]$. Massive gravity has also been employed to define effective field theories for phonons $[9,10]$.

2 The present paper describes phonons corresponding to sliding modes of spatially modulated order parameters, not to be confused with the phonons due to the ionic lattice.
} 
- Spontaneous pattern formation is at the basis of the density waves physics $[19,20]$. Dynamical density waves are the best candidates to explain the anomalous transport properties of many strongly-correlated electron systems (e.g. high- $T_{c}$ superconductors both in the "normal" and "condensed" phases [20]).

- Implementation and check of general expectation about the consequences of the symmetry breaking.

In this latter sense, we prove the validity of Gell Mann-OakesRenner relations for pseudo-phonons: ${ }^{3}$ their squared mass is, at leading order, linear in the perturbation which breaks translations explicitly.

A perturbative explicit component of translation symmetry breaking can model the effects of weak disorder. In particular, it pins a modulated order parameter preventing it from sliding freely. Weakly pinned density waves are the best candidate mechanism to explain the bad metal phenomenology [22-24]. The argument goes as follows: despite having little disorder, bad metals have exceptionally low dc conductivity due to a significant spectral weight transfer to higher frequencies. In other words, the optical conductivity has a marked peak at finite frequency, which subtracts spectral weight from null frequency. The soft finite-frequency peak can have a direct connection to a pseudo-phonon associated to a weakly-pinned, modulated order parameter. This hypothesis is further corroborated by the ubiquitous presence of spatial patterns throughout the phase diagram of stronglycorrelated electron systems [25-27].

The models studied in the present paper shed light on possible field theory duals to holographic models which break translation symmetry homogeneously. ${ }^{4}$ Recall that a global symmetry in the gravity model it is not expected to correspond to a global symmetry in the dual field theory. Specifically, the presence of a bulk conserved current does not imply the presence of a corresponding conserved current in the boundary theory. Nonetheless, when broken by a non-trivial field profile with UV Dirichlet boundary conditions, global bulk symmetries have been observed to produce massless modes in the dual field theory $[32,34]$. Among others, this is a specific open question emerging from holographic Q-lattices which motivates further study with alternative approaches.

\section{Main results}

The main results of the paper are:

\footnotetext{
$\overline{3}$ The word pseudo refers to the spontaneous breaking of an approximate symmetry.

4 The models analyzed in this paper have indeed been suggested by holographic theories, see for instance $[1,28-30]$. In a wider historical perspective, it is fair to acknowledge the Q-ball construction as an inspiring progenitor [31].
}

1. The emergence of phonons in a generic class of field theories (1), and the characterization of their dispersion relation (21).

2. The addition of a perturbative term that breaks translation symmetry explicitly and the appearance of a mass for the phonon (31).

3. Realization of homogeneous translation symmetry breaking in a purely field theoretic model (Sect. 4).

4. Construction of toy-models for the formation of concomitant density waves at an angle (Sect. 6).

\section{Phonon and pseudo-phonon}

\subsection{Spontaneous breaking of translations}

Consider the following action

$$
\begin{aligned}
S= & \int d^{3} x\left\{-\left(\partial^{t} \phi^{*}\right)\left(\partial_{t} \phi\right)+A\left(\partial^{i} \phi^{*}\right)\left(\partial_{i} \phi\right)\right. \\
& -B\left[\left(\partial^{i} \phi^{*}\right)\left(\partial_{i} \phi\right)\right]^{2}-F \phi^{*} \phi\left(\partial^{i} \partial^{j} \phi^{*}\right)\left(\partial_{i} \partial_{j} \phi\right) \\
& \left.+G\left(\partial^{i} \phi^{*}\right)\left(\partial_{i} \phi^{*}\right)\left(\partial^{j} \phi\right)\left(\partial_{j} \phi\right)\right\},
\end{aligned}
$$

for a scalar complex field $\phi$ where $A, B, F$ and $G$ are real positive numbers and $i, j$ are spatial indexes, $i, j=1,2$. The metric signature is $(-1,1,1)$. The terms controlled by the couplings $B, F$ and $G$ are fourth-order both in the spatial derivatives and in the field. It is convenient to parametrize the complex field in terms of the modulus and the phase fields

$\phi(t, x, y)=\rho(t, x, y) e^{i \varphi(t, x, y)}$.

Model (1) is spatially isotropic but breaks Lorentz invariance explicitly, it enjoys spacetime translation invariance and global $U(1)$ phase rotations of $\phi$ (namely shift symmetry for the phase field $\varphi$ ). The Euler-Lagrange equations for $\phi$ are given by

$$
\begin{aligned}
\operatorname{EOM}[\phi]= & \partial^{t} \partial_{t} \phi-A \partial^{i} \partial_{i} \phi+2 B \partial^{i}\left[\partial^{j} \phi^{*} \partial_{j} \phi \partial_{i} \phi\right] \\
& -F \partial^{i} \partial^{j}\left[\phi^{*} \phi \partial_{i} \partial_{j} \phi\right]-F \phi\left(\partial^{i} \partial^{j} \phi^{*}\right)\left(\partial_{i} \partial_{j} \phi\right) \\
& -2 G \partial^{i}\left[\partial_{i} \phi^{*} \partial^{j} \phi \partial_{j} \phi\right]=0,
\end{aligned}
$$

and its complex conjugate equation $\operatorname{EOM}\left[\phi^{*}\right]$. Recall that the variational problem leading to the equations of motion assumes $\delta \phi=\delta \phi^{*}=0$ at infinity. Passing to the "polar representation" (2), one can obtain the equations of motion for the modulus and phase fields by means of the combinations

$\rho \operatorname{EOM}[\rho]=\phi^{*} \operatorname{EOM}[\phi]+\phi \operatorname{EOM}\left[\phi^{*}\right]$, 
$i \operatorname{EOM}[\varphi]=\phi^{*} \operatorname{EOM}[\phi]-\phi \operatorname{EOM}\left[\phi^{*}\right]$.

Consider the following static but $x$-dependent ansatz:

$\rho(t, x, y)=\bar{\rho}, \quad \varphi(t, x, y)=k x$,

and plug it into the modulus equation of motion (4), thus obtaining

$\operatorname{EOM}[\rho]=2 k^{2} \bar{\rho}^{2}\left[A-2 k^{2} \bar{\rho}^{2}(B+F-G)\right]=0$,

while the equation of motion for $\varphi(5)$ is automatically satisfied. The solutions to (7) $\operatorname{are}^{5}$

$k^{(1,2)}=0$,

$k^{(3,4)}= \pm \frac{1}{\bar{\rho}}\left[\frac{A}{2(B+F-G)}\right]^{\frac{1}{2}}$.

To have real solutions we demand $B+F>G$. The energy density of a static configuration of the form (6) is given by

$\mathcal{E}(k, \bar{\rho})=k^{2} \bar{\rho}^{2}\left[-A+k^{2} \bar{\rho}^{2}(B+F-G)\right]$,

and it is spatially homogeneous. In particular, on the solutions (8) and (9) the energy attains the following values

$\mathcal{E}\left(k^{(1,2)}\right)=0$,

$\mathcal{E}\left(k^{(3,4)}\right)=-\frac{1}{2} A\left(k^{(3,4)}\right)^{2} \bar{\rho}^{2}$.

Notice that $\mathcal{E}\left(k^{(3,4)}\right)$ represent degenerate global minima because $A>0$. In Figure 1 we plot a specific example.

Having specified the background of interest (6), let us consider now the fluctuations

$\phi(t, x, y)=[\bar{\rho}+\delta \eta(t, x, y)] e^{i k x}$

and parametrize the complex fluctuation field $\delta \eta$ by its real and imaginary parts

$\delta \eta(t, x, y)=\sigma(t, x, y)+i \tau(t, x, y)$.

The quadratic action for the fluctuations in Fourier space is given by

$S_{(2)}=\int \frac{d^{3} q}{(2 \pi)^{3}} \tilde{v}(-q)^{T} \cdot M \cdot \tilde{v}(q)$,

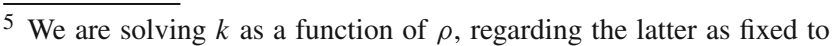
a non-trivial value by boundary conditions. Comments on the handling of boundary conditions are given later, especially in Sect. 5 .
}

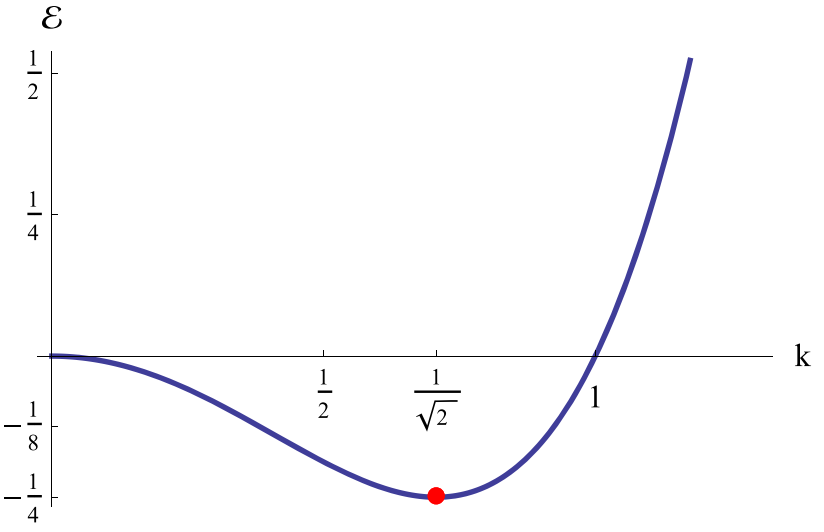

Fig. 1 Plot of the static energy for the particular case $A=B=F=$ $G=\bar{\rho}=1$. The red dot corresponds to the global minimum of the energy, $-1 / 4$, attained for $k=1 / \sqrt{2}$

where $q=\left(\omega, q_{x}, q_{y}\right)$ and

$\tilde{v}(q)=\left(\begin{array}{c}\tilde{\sigma}(q) \\ \tilde{\tau}(q)\end{array}\right)$

The entries of the matrix $M$ defined in (15) are given by

$$
\begin{aligned}
M_{\sigma \sigma}= & \omega^{2}-2 A k^{2}-4 k^{2} \bar{\rho}^{2}\left(2 F q_{x}^{2}+G q_{y}^{2}\right) \\
& -F \bar{\rho}^{2}\left(q_{x}^{2}+q_{y}^{2}\right)^{2}, \\
M_{\tau \tau}= & \omega^{2}-2 A q_{x}^{2}-F \bar{\rho}^{2}\left(q_{x}^{2}+q_{y}^{2}\right)^{2}, \\
M_{\sigma \tau}= & M_{\tau \sigma}^{*}=-2 i k q_{x}\left[A+2 F \bar{\rho}^{2}\left(q_{x}^{2}+q_{y}^{2}\right)\right] .
\end{aligned}
$$

The eigenvalues $e_{1,2}$ of $M$ are given by complicated expressions. To highlight the physical characteristics of the two modes, it is convenient to expand the dispersion relations $e_{1,2}=0$ for low momenta, thus obtaining

$\omega^{2}=2 A k^{2}+4 k^{2} \bar{\rho}^{2}\left[(B+3 F-G) q_{x}^{2}+G q_{y}^{2}\right]+\ldots$,

where $k$ is given by (9), and

$\omega^{2}=\bar{\rho}^{2}\left[2(2 G-3 F) q_{x}^{2} q_{y}^{2}+F\left(q_{x}^{4}+q_{y}^{4}\right)\right]+\ldots$

In (21), the positivity of the quartic term in momenta requires $G \geq F$. We also require $F \neq 0$. Indeed, for $F=0$ there would be neither pure longitudinal $\left(q_{y}=0\right)$ nor pure transverse $\left(q_{x}=0\right)$ propagation in (21). Note that $F \neq 0$ implies that we are keeping in the action (1) a term with more than one spatial derivative acting on a single field. Since a similar term in time derivatives would lead to Ostrogradsky instabilities $[35,36]$, the condition $F \neq 0$ constitutes an obstruction to building simple relativistic generalizations of model (1). 
Combining the requirements above we have

$G \geq F>0$.

Equation (21) describes a gapless mode which is the phonon. ${ }^{6}$ It represents the Nambu-Goldstone mode arising from the spontaneous breaking of the product of phase shifts and translations to its diagonal subgroup. The phonon dispersion relation is not standard, because $\omega$ is not linear in the momentum, ${ }^{7}$ and the propagation is in general not isotropic (despite being $x \leftrightarrow y$ symmetric). The dispersion relation given in (20) corresponds instead to a gapped mode. Both its mass and the leading $q_{x}$ term are not essentially related to the couplings $F$ and $G$, but the transverse propagation at quadratic order would vanish for $G=0$. In general such gapped mode does not propagate isotropically and its dispersion relation is not symmetric under $x \leftrightarrow y$ exchange.

As a final comment, studying the fluctuations about a background (8) with $k=0$, one finds that it is locally unstable. This is a consequence of the gradient Mexican hat Lagrangian (1), which does not admit a stable, unmodulated order parameter. In other words, although the model bears close resemblance with a standard superfluid, the presence of a non-trivial condensate requires necessarily a non-trivial $k$ and, thereby, a linear spatial variation of the phase. As noted below (37), the current density on solutions (6) of model (1) is zero despite $k$ being non-null.

\subsection{Adding a small explicit translation breaking}

We add to the action (1) a perturbation which breaks translations along $x$ explicitly:

$$
\begin{aligned}
S= & \int d^{3} x\left\{-\left(\partial^{t} \phi^{*}\right)\left(\partial_{t} \phi\right)+A\left(\partial^{i} \phi^{*}\right)\left(\partial_{i} \phi\right)\right. \\
& -B\left[\left(\partial^{i} \phi^{*}\right)\left(\partial_{i} \phi\right)\right]^{2}-F \phi^{*} \phi\left(\partial^{i} \partial^{j} \phi^{*}\right)\left(\partial_{i} \partial_{j} \phi\right) \\
& \left.+G\left(\partial^{i} \phi^{*}\right)\left(\partial_{i} \phi^{*}\right)\left(\partial^{j} \phi\right)\left(\partial_{j} \phi\right)+n f_{\kappa}\left(x, \phi^{*}, \phi\right)\right\},
\end{aligned}
$$

where $n$ is a perturbative coupling, and $f_{\kappa}$ is an explicit symmetry-breaking term controlled by the parameter $\kappa$. Concretely we take:

$f_{\kappa}\left(x, \phi^{*}, \phi\right)=\frac{\kappa^{2}}{2}\left[\phi e^{-i \kappa x}-\phi^{*} e^{i \kappa x}\right]^{2}$,

which vanishes for $\kappa \rightarrow 0$.

\footnotetext{
6 We use the term phonon to indicate the Nambu-Goldstone mode arising from the spontaneous breaking of spatial translations.

7 It is interesting to note that a quadratic dispersion relation was obtained for the scalar mode of the ghost condensate [12] too. In the literature one can encounter other examples of phonons with a quadratic dispersion relation [14].
}

We consider again the ansatz (6), this time the equation of motion (5) for the phase field $\varphi$ is not automatically satisfied, but takes the form

$\operatorname{EOM}[\varphi]=-2 i n \kappa^{2} \bar{\rho}^{2} \sin [2 x(k-\kappa)]$.

To solve (25) we fix

$k=\kappa$.

Upon considering (26), the equation of motion for the modulus field $\rho$ reduces again to (4).

The quadratic action for the fluctuations gets modified by the perturbation, in particular the entries of the matrix $M$ introduced in (15) become

$$
\begin{aligned}
M_{\sigma \sigma}= & \omega^{2}-2 A k^{2}-4 k^{2} \bar{\rho}^{2}\left(2 F q_{x}^{2}+G q_{y}^{2}\right) \\
& -F \bar{\rho}^{2}\left(q_{x}^{2}+q_{y}^{2}\right)^{2}, \\
M_{\tau \tau}= & \omega^{2}-2 n \kappa^{2}-2 A q_{x}^{2}-F \bar{\rho}^{2}\left(q_{x}^{2}+q_{y}^{2}\right)^{2}, \\
M_{\sigma \tau}= & M_{\tau \sigma}^{*}=-2 i k q_{x}\left[A+2 F \bar{\rho}^{2}\left(q_{x}^{2}+q_{y}^{2}\right)\right] .
\end{aligned}
$$

The only difference with respect to the spontaneous case is given by a new $-2 n \kappa^{2}$ term in $M_{\tau \tau}$. Expanding the two dispersion relations $e_{1,2}=0$ in low momenta and in small $n$, one obtains ${ }^{8}$

$$
\begin{aligned}
\omega^{2}= & 2 A k^{2}+2 n q_{x}^{2} \\
& +4 k^{2} \bar{\rho}^{2}\left[(B+3 F-G) q_{x}^{2}+G q_{y}^{2}\right]+\ldots,
\end{aligned}
$$

and

$$
\begin{aligned}
\omega^{2} & =2 n \kappa^{2}-2 n q_{x}^{2}+\left[F+\frac{4}{A}(B+3 F-G) n\right] \bar{\rho}^{2} q_{x}^{4} \\
& +2\left[(2 G-3 F)+\frac{4}{A}(G-F) n\right] \bar{\rho}^{2} q_{x}^{2} q_{y}^{2}+F \bar{\rho}^{2} q_{y}^{4}+\ldots,
\end{aligned}
$$

where $k$ and $\kappa$ are given by (26) and (9). The squared mass $2 n \kappa^{2}$ in (31) is linear in the perturbative coupling $n$ which controls the explicit breaking, this agrees with Gell MannOakes-Renner expectation for a pseudo Nambu-Goldstone mode, thereby (31) describes a pseudo-phonon..$^{9}$ Note also that the explicit breaking affects both the dispersion relations of the gapped and the Nambu-Goldstone modes at the quadratic level in $q_{x}$. This is particularly relevant for the pseudo-phonon as it lowers the leading order at which the longitudinal momentum enters the dispersion relation. As

\footnotetext{
$\overline{8}_{\text {Recall that } e_{1,2}}$ are the eigenvalues of the matrix $M$.

${ }^{9}$ Gell Mann-Oakes-Renner relations for phonons were obtained in holographic models in [28]. For an experimental study of gapped sliding modes, see for example [21].
} 
far as the leading transverse propagation is concerned, the explicit breaking term does not alter the qualitative picture.

\section{Comments on homogeneous translation symmetry breaking}

A solution of the form (6) breaks the product of translations along $x$ and $\varphi$-shifts to the diagonal subgroup, so a translation along $x$ can be compensated by a global phase shift. This is the hallmark of homogeneous translation symmetry breaking: any spacetime point is equivalent to any other up to a global internal transformation. There is no unit cell and $k$ should not be strictly speaking interpreted as the wave vector of a lattice. ${ }^{10}$

The canonical energy-momentum tensor of model (1) is given by

$$
\begin{aligned}
T^{\mu \nu} & =\frac{1}{2} \eta^{\mu \nu} \mathcal{L}+\delta_{t}^{\mu} \partial^{t} \phi^{*} \partial^{v} \phi+\delta_{i}^{\mu}\left[A \partial^{i} \phi^{*}\right. \\
- & 2 B \partial^{j} \phi^{*} \partial_{j} \phi \partial^{i} \phi^{*}+2 G \partial^{j} \phi^{*} \partial_{j} \phi^{*} \partial^{i} \phi \\
- & \left.F \phi^{*} \phi \partial^{i} \partial^{j} \phi^{*} \partial_{j}+F \partial_{j}\left(\phi^{*} \phi \partial^{i} \partial^{j} \phi^{*}\right)\right] \partial^{v} \phi+\text { c.c. } .
\end{aligned}
$$

The momentum density vanishes $T^{t x}=T^{t y}=0$ on a solution of the form (6). On the equations of motion we have $\partial_{\mu} T^{\mu \nu}=0$, thus the Ward-Takahashi identities for translations are satisfied.

On a solution (6) the energy density is given by

$\epsilon=T^{t t}=\eta^{t t} L_{0}=-L_{0}$,

where $L_{0}$ is the Lagrangian density written in (1) considered on the background (6). Similarly, the spatial components of the energy-momentum tensor are:

$T^{x x}=\eta^{x x} L_{0}+2 \bar{\rho}^{2} k^{2}\left[A-2 \bar{\rho}^{2} k^{2}(B-G+F)\right]=L_{0}$,

$T^{y y}=\eta^{y y} L_{0}=L_{0}$,

where in the second step of (34) we have used the equation of motion (7). We define the pressure $p=-\Omega / V$ where $\Omega / V$ is the Landau potential density. Given that $T=\mu=0$, we have $\Omega / V=\epsilon-T s-\mu n=\epsilon$. Comparing (33) with (34) and (35) we obtain $p=T^{x x}=T^{y y}$. The pressure is thus isotropic and the equation of state is given by ${ }^{11}$

$\epsilon=-p$

\footnotetext{
${ }^{10}$ Relatedly, holographic models with backgrounds that break translations homogeneously do not feature commensurability effects for the formation of stripes [11].

11 This is the same equation of state found for the ghost condensate [12].
}

The $U(1)$ current density is given by

$$
\begin{aligned}
J^{\mu}= & i \delta_{t}^{\mu} \partial^{t} \phi^{*} \phi-i \delta_{i}^{\mu}\left\{A \partial^{i} \phi^{*} \phi-2 B \partial^{i} \phi^{*} \partial^{j} \phi^{*} \partial_{j} \phi \phi\right. \\
& +2 G \partial^{i} \phi \partial^{j} \phi^{*} \partial_{j} \phi^{*} \phi-F \phi^{*} \phi \partial^{i} \partial^{j} \phi^{*} \partial_{j} \phi \\
& \left.+F \partial_{j}\left[\phi^{*} \phi \partial^{i} \partial^{j} \phi^{*}\right] \phi\right\}+ \text { c.c. },
\end{aligned}
$$

and, on a solution (6) of the equation of motion (7), it vanishes too.

Remarkably, the explicit breaking term (24) does not introduce a source into the translation 1-point Ward identity. Indeed, consider the $\phi$ field transformation under a diffeomorphism

$\delta_{\xi} \phi=\xi^{\mu} \partial_{\mu} \phi$

and take $\xi^{\mu}=\delta_{x}^{\mu} \xi$. One has that the explicit breaking term in (23) transforms as follows:

$$
\begin{aligned}
& \delta_{\xi} f_{\kappa}\left(x, \phi^{*}, \phi\right)=\frac{\delta f_{\kappa}}{\delta \phi} \delta_{\xi} \phi+\frac{\delta f_{\kappa}}{\delta \phi^{*}} \delta_{\xi} \phi^{*} \\
& =\kappa^{2} \xi\left[\phi e^{-i \kappa x}-\phi^{*} e^{i \kappa x}\right]\left[e^{-i \kappa x} \partial_{x} \phi-e^{i \kappa x} \partial_{x} \phi^{*}\right] \\
& =2 i \kappa^{3} \xi \bar{\rho}(\bar{\rho}-\bar{\rho})=0,
\end{aligned}
$$

where in the last passages we have used both the ansatz (6) and the condition (26). The triviality of the 1-point WardTakahashi identity for translations, in spite of the presence of a term which breaks translations explicitly, is a peculiarity of homogeneous breakings. These have already been studied in holographic models, see [28] for instance.

Analogous arguments show that the explicit term (24) does not introduce a source into the Ward-Takahashi identity of the $U(1)$ symmetry either.

\section{Adding a potential $V\left(\phi^{*} \phi\right)$}

Consider the action (23) with the addition of a generic potential term respecting the global $U(1)$ symmetry,

$$
\begin{aligned}
S= & \int d^{3} x\left\{-\left(\partial^{t} \phi^{*}\right)\left(\partial_{t} \phi\right)+A\left(\partial^{i} \phi^{*}\right)\left(\partial_{i} \phi\right)\right. \\
& -B\left[\left(\partial^{i} \phi^{*}\right)\left(\partial_{i} \phi\right)\right]^{2}-F \phi^{*} \phi\left(\partial^{i} \partial^{j} \phi^{*}\right)\left(\partial_{i} \partial_{j} \phi\right) \\
& +G\left(\partial^{i} \phi^{*}\right)\left(\partial_{i} \phi^{*}\right)\left(\partial^{j} \phi\right)\left(\partial_{j} \phi\right) \\
& \left.+n f_{\kappa}\left(x, \phi^{*}, \phi\right)-V\left(\phi^{*} \phi\right)\right\} .
\end{aligned}
$$

Apart from providing a more generic situation, a potential $V\left(\phi^{*} \phi\right)$ is important for a specific reasons: as shown later, the dispersion relation of the phonon becomes in general linear in $q_{x}$ when a potential $V\left(\phi^{*} \phi\right)$ is considered. 
The explicit breaking term $n f_{\kappa}\left(x, \phi^{*}, \phi\right)$ in (40) still requires $\kappa=k$, which in turn solves $\operatorname{EOM}[\varphi]=0$ automatically. The equation of motion (7) for the modulus field gets instead modified into

$2 \bar{\rho}^{2}\left\{k^{2}\left[A-2 k^{2} \bar{\rho}^{2}(B+F-G)\right]-V^{\prime}\left(\bar{\rho}^{2}\right)\right\}=0$.

The energy is given by

$\mathcal{E}(k, \bar{\rho})=k^{2} \bar{\rho}^{2}\left[-A+k^{2} \bar{\rho}^{2}(B+F-G)\right]+V\left(\bar{\rho}^{2}\right)$,

and its minimization with respect to $\bar{\rho}$ and $k$ returns respectively the equation of motion (41) and the extra condition

$2 k \bar{\rho}^{2}\left[2(B+F-G) k^{2} \bar{\rho}^{2}-A\right]=0$.

The composition of the equation of motion (41) with condition (43) implies

$V^{\prime}\left(\bar{\rho}^{2}\right)=0$

Studying the current density (37) in the presence of a nontrivial potential $V$, it is possible to show that condition (44) corresponds to asking for a vanishing background current density and strain.

A variation $\delta k$ in (6) corresponds to $\delta \varphi=\delta k x$, which does not vanish for asymptotically large $|x|$, it even diverges. In particular, it lies outside of the hypothesis assumed for the variational problem to derive the equations of motion. One can nevertheless take $\delta k$ variations and derive (43) relying on a regularization of the system to a finite box ${ }^{12}$ and focusing exclusively on the dynamical character of the symmetry breaking. Said otherwise, one can neglect the boundary term in the variation of the action or, equivalently, assume that the boundary conditions be free. Since the minimization with respect to $\delta \bar{\rho}$ alone already reproduces the only non-trivial equation of motion (41), the study of the energy minimization with respect to both $\delta \bar{\rho}$ and $\delta k$ selects a subset among the solutions to (41) (considered with free boundary conditions).

The entries of the matrix $M$ (defined in (15)) for the quadratic fluctuation action become

$$
\begin{aligned}
M_{\sigma \sigma}= & \omega^{2}-2 A k^{2}-4 k^{2} \bar{\rho}^{2}\left(2 F q_{x}^{2}+G q_{y}^{2}\right) \\
& -F \bar{\rho}^{2}\left(q_{x}^{2}+q_{y}^{2}\right)^{2}-2 \bar{\rho}^{2} V^{\prime \prime}\left(\bar{\rho}^{2}\right), \\
M_{\tau \tau}= & \omega^{2}-2 n \kappa^{2}-2 A q_{x}^{2}-F \bar{\rho}^{2}\left(q_{x}^{2}+q_{y}^{2}\right)^{2}, \\
M_{\sigma \tau}= & M_{\tau \sigma}^{*}=-2 i k q_{x}\left[A+2 F \bar{\rho}^{2}\left(q_{x}^{2}+q_{y}^{2}\right)\right] .
\end{aligned}
$$

\footnotetext{
12 Comments on how to define Nambu-Goldstone bosons in finite-size systems are given in [42].
}

where (44) has been already considered, so we are taking fluctuations over a background that satisfies the energy minimization condition (43).

The eigenvalues $e_{1,2}$ of $M$ yield the dispersion relations of two modes through $e_{1,2}=0$. Let us expand these dispersion relations in low momenta and in small $n$ :

$$
\begin{aligned}
\omega^{2} & =2 A k^{2}+2 \bar{\rho}^{2} V^{\prime \prime}\left(\bar{\rho}^{2}\right)+\frac{2 A^{4} n q_{x}^{2}}{\left(A^{2}+2 H \bar{\rho}^{4} V^{\prime \prime}\left(\bar{\rho}^{2}\right)\right)^{2}} \\
& +4 G k^{2} \bar{\rho}^{2} q_{y}^{2}+\frac{4 A \bar{\rho}^{2} q_{x}^{2}\left[A(2 F+H) k^{2}+2 F \bar{\rho}^{2} V^{\prime \prime}\left(\bar{\rho}^{2}\right)\right]}{A^{2}+2 H \bar{\rho}^{4} V^{\prime \prime}\left(\bar{\rho}^{2}\right)}+\ldots
\end{aligned}
$$

and

$$
\begin{aligned}
\omega^{2}= & 2 n \kappa^{2}+\frac{4 A H \bar{\rho}^{4} V^{\prime \prime}\left(\bar{\rho}^{2}\right) q_{x}^{2}}{A^{2}+2 H \bar{\rho}^{4} V^{\prime \prime}\left(\bar{\rho}^{2}\right)} \\
& -\frac{4 A^{3} H \bar{\rho}^{2} n \kappa^{2} q_{x}^{2}}{\left[A^{2}+2 H \bar{\rho}^{4} V^{\prime \prime}\left(\bar{\rho}^{2}\right)\right]^{2}}+\ldots
\end{aligned}
$$

Where again $\kappa$ and $k$ are given by (26) and (9), we have introduced the positive quantity (see comment below (9))

$H=B+F-G$,

and we assumed $V^{\prime \prime}\left(\bar{\rho}^{2}\right)>0$, in accordance with a positive concavity for the energy (42) with respect to $\delta \bar{\rho}$ variations.

The potential $V\left(\phi^{*} \phi\right)$ does not alter the overall qualitative picture already obtained in Sect. 3.2; the dispersion relations (48) and (49) still describe respectively a gapped mode and a pseudo-phonon with an $n$-linear squared mass. Note however that $V\left(\phi^{*} \phi\right)$ would make a qualitative difference in the purely spontaneous case: if $n=0$, the leading $q_{x}$ term in the dispersion relation of the phonon is either quadratic or quartic depending on $V^{\prime \prime}\left(\bar{\rho}^{2}\right)$ being trivial or not. The phonon dispersion relation is

$\omega=c_{\mathrm{ph}} q_{x}+O\left(q_{x}^{2}\right)$

where the longitudinal speed of propagation $c_{\mathrm{ph}}$ is proportional $V^{\prime \prime}\left(\bar{\rho}^{2}\right)$. Importantly, one can repeat the analysis of Sect. 4 also in the presence of $V\left(\phi^{*} \phi\right)$, still getting the same equation of state $\epsilon=-p$ obtained in (36). ${ }^{13}$ Specifically, the on-shell value of the Lagrangian density $L_{0}$ is affected by the potential, but Eqs. (33), (34) and (35) are still valid.

\footnotetext{
${ }^{13}$ In the presence of $V\left(\phi^{*} \phi\right)$, one needs to consider the equation of motion (41) and the minimization condition (43). An analogous result was found in [13] for modulated holographic phases where, despite the presence spontaneous modulations, the equilibrium stress-energy tensor assumes the perfect fluid form.
} 


\section{Comments on lattice toy models}

We still consider a setup with two spatial directions, $x$ and $y$, and construct vacua with two coexisting space-dependent configurations (each one similar to that of Sect. 3) characterized by two vectors $\mathbf{k}_{1}$ and $\mathbf{k}_{2}$. To this purpose we need two independent scalar fields, $\phi_{1}$ and $\phi_{2}$. We consider doubling the model (40) and adding a cross-term whose role is to choose vacua where $\mathbf{k}_{1}$ and $\mathbf{k}_{2}$ form a specific angle $\theta$; namely $\mathbf{k}_{1} \cdot \mathbf{k}_{2}=\left|\mathbf{k}_{1}\right|\left|\mathbf{k}_{2}\right| \cos (\alpha)$ and we want to realize $\alpha=\theta$ dynamically. Calling $L\left[\phi^{*}, \phi\right]$ the Lagrangian in (40) with $n=0$, we consider

$$
\begin{aligned}
S_{\theta}= & \int d^{3} x\left\{L\left[\phi_{1}^{*}, \phi_{1}\right]+L\left[\phi_{2}^{*}, \phi_{2}\right]\right. \\
& \left.-\lambda\left|\partial_{i} \phi_{1}^{*} \partial_{j} \phi_{2}^{*}\left(\partial^{i} \phi_{2} \partial^{j} \phi_{1}-\cos ^{2}(\theta) \partial^{i} \phi_{1} \partial^{j} \phi_{2}\right)\right|^{2}\right\} .
\end{aligned}
$$

The term in $\lambda$ leads in fact to the minimization of

$$
\begin{aligned}
& \left(\mathbf{k}_{1} \cdot \mathbf{k}_{2}\right)^{2}-\left|\mathbf{k}_{1}\right|^{2}\left|\mathbf{k}_{2}\right|^{2} \cos ^{2}(\theta) \\
& \quad=\left|\mathbf{k}_{1}\right|^{2}\left|\mathbf{k}_{2}\right|^{2}\left[\cos ^{2}(\alpha)-\cos ^{2}(\theta)\right] .
\end{aligned}
$$

For field configurations where $\mathbf{k}_{1}$ and $\mathbf{k}_{2}$ form an angle $\theta$, the term in $\lambda$ vanishes and the energy of the configuration is the same as that of two non-interacting space-dependent condensates (i.e. $\lambda=0$ ). Since the $\lambda$ term is the square of a quantity that vanishes on the background, also at the level of linear fluctuations there are no effects due to $\lambda$. In particular, the stability analysis of the background is not affected by $\lambda$.

Model (52) enjoys a global $U(1) \times U(1)$ symmetry and leads to vacua where $\left|\mathbf{k}_{1}\right|=\left|\mathbf{k}_{2}\right|$. It can however be generalized to cases with a smaller symmetry. As far as the $\lambda$ term is concerned, there is an interesting alternative to (52),

$-\lambda\left|\partial_{i} \phi_{1}^{*} \partial_{j} \phi_{2}\left(\partial^{i} \phi_{2}^{*} \partial^{j} \phi_{1}-\cos ^{2}(\theta) \partial^{i} \phi_{1}^{*} \partial^{j} \phi_{2}\right)\right|^{2}$,

which preserves only a global $U(1)$ symmetry and still leads to the minimization of (53). The phases of the two fields are locked and it is no longer true that a global internal symmetry transformation can compensate for a generic translation $\Delta \mathbf{x}$. This is a way to partially breaking the homogeneity down to transformations satisfying

$\mathbf{k}_{1} \cdot \Delta \mathbf{x}=\mathbf{k}_{2} \cdot \Delta \mathbf{x}=-\Delta \varphi$.

\section{Discussion and future directions}

The models studied in this paper can be generalized to spacetimes with higher dimensionality. The addition of transverse spatial directions does not affect the essential aspects of the computations and the features of the low-energy modes. ${ }^{14}$

\subsection{Need for higher derivatives}

We pursued the spontaneous breaking of translation symmetry following a "Mexican hat" strategy applied to spatial gradients, which implies that quartic terms in the spatial gradients are a necessary ingredient. This is apparent already from the equation of motion (7) where, upon setting $B=F=G=0$, only trivial solutions with either $k=0$ or $\bar{\rho}=0$ remain. Studying the fluctuations about a background (6) with $n=0$ and $V\left(\phi^{*} \phi\right)=0$, the Nambu-Goldstone frequency does not feature independent longitudinal and transverse propagation unless the coupling $F$ is non-trivial, see (21).

To the purpose of finding the simplest possible models, we restrained the attention to terms whose order in spatial derivatives equals the order in the fields. ${ }^{15}$ There are three observations about this: (i) it is remarkable that in order to obtain a propagating phonon the Lagrangian in (1) needs to be already quite complicated; (ii) it would be interesting to repeat the present analysis allowing for all the possible consistent terms. ${ }^{16}$ (iii) Higher spatial derivatives are analogous to frustration. ${ }^{17}$

The $F$ term in (1) features more than one derivative applied on the same field. This could not be avoided even considering spatial partial integrations. Such a term, if covariantized,

\footnotetext{
14 The models could actually be considered also in $1+1$ dimensions, suppressing the $y$ direction. Still, the essential computational points about the pseudo Nambu-Goldstone modes and propagation along $x$ would remain unaltered. On the interpretation level there are however extra subtleties: spontaneous symmetry breaking in $1+1$ dimensions is impeded by Coleman-Mermin-Wagner-Hohenberg theorem. Such obstruction is avoided by quantum field theories in the large $N$ limit, roughly because the fluctuations which would spoil the condensation of the order parameter are suppressed. It would be interesting to consider whether, already in $1+1$ dimensions, the models studied here could be regarded as effective descriptions of theories in the strict large $N$ limit. Two relevant references on these points are: [41] where the details of symmetry breaking in (large $N$ ) holographic field theories in $1+1$ dimensions have been analyzed; and [42] which argues on the existence of Nambu-Goldstone bosons in $1+1$ dimensions, even without a strict spontaneous symmetry breaking.

15 In [17] a similar claim is made in relation to generic effective field theories for phonons. Comments on power-counting schemes for theories enjoying shift symmetries are given in [18].

${ }^{16}$ In model (1), the order 4 for spatial derivatives and 2 for temporal ones can be argued by assuming $z=2$ non-relativistic scaling for the time coordinate and time-reversal invariance.

${ }^{17}$ In discretized theories a modulated vacuum arises typically because of the competition between different couplings, e.g. the next-neighbor coupling competing with the next-to-next-neighbor coupling. If one discretizes the model (1), something similar would happen, for instance the next-to-next-neighbor coupling due to the $F$ term competes with the next-neighbor coupling due to the $A$ term.
} 
would lead to multiple time derivatives applied on the same field, which in turn would produce Ostrogradsky instabilities $[35,36]$. As a consequence, the models studied in this paper do not admit a trivial relativistic generalization.

The possibility of breaking translations spontaneously in relativistic theories is interesting in relation to the systematic approach of [17] which classifies all theoretically possible condensed matter systems in terms of their spontaneous breaking of Poincaré invariance. ${ }^{18}$ The models of the present paper could possibly be thought of as effective low-energy descriptions of relativistic UV theories, assuming that a spontaneous Lorentz symmetry breaking has occurred along the renormalization group flow at a scale above the cutoff of the effective theory. Studying the possibility of such completions is a future direction. The embedding of the present models into a systematic effective field theory framework is a future perspective too. To this regard one could, or perhaps should, consider topological terms in the action and couplings with generic dependence on $\phi$.

\subsection{The role of boundary conditions}

In the model (40), which features a potential $V\left(\phi^{*} \phi\right)$, it proved to be essential to minimize the energy with respect to both the parameters of the ansatz (6), $k$ and $\bar{\rho}$. This amounts to minimizing the energy without fixing the boundary conditions for the field $\phi$. It is important to recall that the apparent larger freedom implied by relaxing the boundary conditions is actually compensated by an additional requirement on the solutions: the extra condition descending from the energy minimization with respect to variations of $k$.

In order to stress the role played by the boundary conditions we can consider a somewhat complementary example: a kinetic symmetry breaking where the translations are actually broken by boundary conditions. ${ }^{19}$ Let us take the simple example of a free real scalar field whose Lagrangian is just $\partial_{\mu} \psi \partial^{\mu} \psi$. This scalar field can be intuitively related to the phase field $\varphi$ in (6) and in fact one can consider an ansatz $\psi=k x$. The equation of motion is satisfied for any value of $k$. To avoid problems with diverging fields, one can regularize the space to a finite box and impose boundary conditions that are compatible with the ansatz for a specific value of $k$. Said otherwise, if the boundary conditions are fixed, they dictate the value of $k$ regardless of energy considerations. ${ }^{20}$ There is a translation symmetry breaking, but it is forced

\footnotetext{
$\overline{18}$ The generation of spatially modulated vacua in relativistic field theories has been considered in [37].

19 See [38] which contains a similar discussion in relation to kink solutions induced by boundary conditions.

${ }^{20}$ If instead the boundary conditions are free, the value of $k$ is arbitrary.
}

by a "kinetic" constraint instead of being generated dynamically. ${ }^{21}$

\subsection{Topologically non-trivial configurations}

The gradient Mexican hat potential discloses various possibilities to construct non-trivial topological objects. We briefly comment some instances, which are however regarded as future prospects.

A possible topologically non-trivial configuration is the gradient kink. When the "potential" for gradients allows for degenerate absolute minima (e.g. the solutions (8)), there are sectors where the solutions feature a jump in $k$ necessary to connect two degenerate minima. If the direction of the gradient is along the direction of the step profile, the gradient kink is longitudinal and can be mapped to a normal kink by redefining the gradient field as the fundamental field, $\partial_{x} \phi \rightarrow$ $\psi$.

Another slightly more exotic possibility is to combine the topological non-triviality in the gradient sector and that resulting from possible degenerate minima in the potential $V\left(\phi^{*} \phi\right)$.

As a final remark, the structure of the the models studied in the present paper, and specifically the fact that they contain terms with different signs and different scaling properties under spatial dilatations, makes them avoid Derrick's no-go theorem for the existence of finite energy solitons [40].

\subsection{Comments on phenomenology: transport, sound and helical orderings}

The models studied here can be embedded in larger theories and provide sub-sectors where translations are broken spontaneously or pseudo-spontaneously. The phenomenological properties of the larger system, like for example transport, would however depend on the coupling of the translation breaking sub-sector with the larger system and, of course, on the characteristics of the latter itself.

One cannot study the compressional sound mode from the equation of state (36) because neither $\epsilon$ nor $p$ depend on the volume. ${ }^{22}$ The first law of thermodynamics actually coincides with the equation of state (36). Remarkably, these comments hold independently of the presence of a potential $V\left(\phi^{*} \phi\right)$, see Sect. 5. The sound mode is instead described

\footnotetext{
${ }^{21}$ The simplicity of the free real scalar $\psi$ just described is deceitful: being quadratic in the fields, the model presents a gapless excitation even when the breaking is kinetic, such gapless mode however is not present in more general theories that feature kinetic translation breaking; in particular this gapless mode is not a Nambu-Goldstone because the breaking is not dynamical.

22 An analogous equation of state has been studied in [56] and claimed to be consistent with a vanishing speed of sound. Quintessence models have similar equations of states too, see [57].
} 
upon interpreting the real field $\rho \sin (\varphi-k x)$ whose fluctuation is given by $\tau$ (introduced in (14)) as a displacement field in a target space. Since the model contains higher derivatives terms, one cannot just adopt the standard formulae for the speed of sound in terms of the elastic modulus. One cannot either adopt hydrodynamic formulae, as the system at hand is at zero temperature and zero density. Rather, the sound mode is studied by the fluctuation analysis of the quadratic action, as described in previous sections.

As a possible future application, it would be interesting to gain intuition on the qualitative behavior of holographic low-energy modes by means of a purely field theoretical toymodel. ${ }^{23}$ This is especially interesting in order to match the finite temperature hydrodynamic modes to those of an appropriate $T=0$ theory.

Eventually, the models at hand are technically similar to helical orderings studied in ferromagnetic systems [58]. ${ }^{24}$ There the frequency of the spin-wave modes has been claimed to depend linearly on the longitudinal momentum and quadratically on the transverse momentum [59], as in (49) above.

Acknowledgements This work has been funded by the Spanish grants FPA2014-52218-P and FPA2017-84436-P by Xunta de Galicia (GRC2013-024), by FEDER and by the María de Maeztu Unit of Excellence MDM-2016-0692

Special thanks go to Riccardo Argurio for fundamental feedback and suggestions throughout the development of the project.

I want to acknowledge Cristoph Adam, Andrea Amoretti, Daniel Areán, Maximilian Attems, Matteo Bertolini, Lorenzo Calibbi, Paolo Creminelli, Davide Forcella, Blaise Goutéraux, Carlos Hoyos, Nicola Maggiore, Javier Mas, Andrea Mezzalira, Giorgio Musso, Alfonso Ramallo, Anibal Sierra-García and Douglas Wertepny for very useful and interesting discussions. The paper is dedicated to the memory of Rosetta Cervetto.

Data Availability Statement This manuscript has no associated data or the data will not be deposited. [Authors' comment: Data sharing not applicable to this article as no datasets were generated or analysed during the current study.]

Open Access This article is distributed under the terms of the Creative Commons Attribution 4.0 International License (http://creativecomm ons.org/licenses/by/4.0/), which permits unrestricted use, distribution, and reproduction in any medium, provided you give appropriate credit to the original author(s) and the source, provide a link to the Creative Commons license, and indicate if changes were made.

Funded by SCOAP ${ }^{3}$.

\section{A Related literature}

Some useful references related to the present paper are:

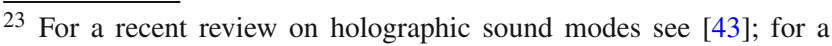
description of a holographic model closer to the field theories of the present paper we refer to [44].

${ }^{24}$ Or helical orderings in ${ }^{3} \mathrm{He}[60]$.
}

- An analysis of pseudo Nambu-Goldstone bosons based on the study of Ward-Takahashi identities has been performed for a $U(1)$ symmetry in a generic quantum field theory in [45], with also a description of its holographic implementation. Similar analyses in a non-relativistic context are performed in $[46,47]$.

- The study of spatial pattern formation is intimately related to the study of phase transitions from a translationally invariant fluid to a solid. We refer to the study of Landau which opened the field [48-50] and to two recent papers relying on similar techniques $[64,65]$. The branch of research related to stripe and density wave formation is huge too, for a review paper aiming at a systematic organization in the context of the copper-oxides we refer to [20].

- The problem of counting theorems for Nambu-Goldstone bosons in general circumstances entailing spacetime symmetries and non-relativistic contexts has gathered recent interest, see for instance [16,51-54].

- The spontaneous locking of internal and external symmetries as the central mechanism for studying effective field theories through coset constructions is described for instance in [17,55].

- The interactions between phonons are dictated by the symmetry breaking, this point has been studied in [15]. It is noteworthy that [15] claims that phonon selfinteraction terms are necessarily present on the basis of consistency and the current algebra. As shown in the main text, a similar conclusion emerges also from the description of the translation symmetry breaking dynamics. A precise comparison among these two claims is left for the future.

- The Q-lattice strategy has been exploited in holography also to address time-dependent systems [61,62]. It would be interesting to consider whether suitable modifications of the models described in the present paper could allow one to study time translations in analogy to spatial translations.

- For a pedagogic review on effective field theories with phonons interacting with electrons see [63].

\section{References}

1. A. Donos, J.P. Gauntlett, JHEP 1404, 040 (2014). https://doi.org/ 10.1007/JHEP04(2014)040. arXiv:1311.3292 [hep-th]

2. T. Andrade, B. Withers, JHEP 1405, 101 (2014). https://doi.org/ 10.1007/JHEP05(2014)101. arXiv:1311.5157 [hep-th]

3. A. Donos, B. Goutéraux, E. Kiritsis, JHEP 1409, 038 (2014). https://doi.org/10.1007/JHEP09(2014)038. arXiv:1406.6351 [hep-th]

4. D. Vegh,. arXiv:1301.0537 [hep-th]

5. R.A. Davison, Phys. Rev. D 88, 086003 (2013). https://doi.org/10. 1103/PhysRevD.88.086003. arXiv:1306.5792 [hep-th] 
6. A. Amoretti, A. Braggio, N. Maggiore, N. Magnoli, D. Musso, JHEP 1409, 160 (2014). https://doi.org/10.1007/ JHEP09(2014)160. arXiv:1406.4134 [hep-th]

7. C. de Rham, G. Gabadadze, A.J. Tolley, Phys. Lett. B 711, 190 (2012). https://doi.org/10.1016/j.physletb.2012.03.081. arXiv:1107.3820 [hep-th]

8. M. Baggioli and O. Pujolás, Phys. Rev. Lett. 114 (2015) no.25, 251602 10.1103/PhysRevLett.114.251602 arXiv:1411.1003 [hepth]

9. L. Alberte, M. Ammon, A. Jiménez-Alba, M. Baggioli, O. Pujolás, Phys. Rev. Lett. 120(17), 171602 (2018). https://doi.org/10.1103/ PhysRevLett.120.171602. arXiv:1711.03100 [hep-th]

10. L. Alberte, M. Baggioli, V.C. Castillo, O. Pujolás,. arXiv:1807.07474 [hep-th]

11. T. Andrade, A. Krikun, JHEP 1605, 039 (2016). https://doi.org/10. 1007/JHEP05(2016)039. arXiv:1512.02465 [hep-th]

12. N. Arkani-Hamed, H.C. Cheng, M.A. Luty, S. Mukohyama, JHEP 0405, 074 (2004). https://doi.org/10.1088/1126-6708/2004/ 05/074. [hep-th/0312099]

13. A. Donos, J .P. Gauntlett, JHEP 1603, 148 (2016). https://doi.org/ 10.1007/JHEP03(2016)148. arXiv:1512.06861 [hep-th]

14. J. Carrete, W. Li, M.L. Lindsay, D. Broido, L. Gallego, N. Mingo, Mater. Res. Lett. 4, 4 (2016). https://doi.org/10.1080/21663831. 2016.1174163. [cond-mat.mtrl-sci/1601.02884]

15. H. Leutwyler, Helv. Phys. Acta 70, 275 (1997). [hep-ph/9609466]

16. I. Low, A.V. Manohar, Phys. Rev. Lett. 88, 101602 (2002). https:// doi.org/10.1103/PhysRevLett.88.101602. [hep-th/0110285]

17. A. Nicolis, R. Penco, F. Piazza, R. Rattazzi, JHEP 1506, 155 (2015). https://doi.org/10.1007/JHEP06(2015)155. arXiv:1501.03845 [hep-th]

18. D.T. Son, M. Wingate, Ann. Phys. 321, 197 (2006). https://doi.org/ 10.1016/j.aop.2005.11.001. [cond-mat/0509786]

19. G. Gruner, Rev. Mod. Phys. 60, 1129 (1988). https://doi.org/10. 1103/RevModPhys.60.1129

20. E. Fradkin, S.A. Kivelson, J.M. Tranquada, Rev. Mod. Phys. 87, 2 (2015). https://doi.org/10.1103/RevModPhys.87.457

21. X. Chen, H.D. Bansal, S. Sullivan, D. Abernathy, A. Aczel, J. Zhou, O. Delaire, L. Shi, Phys. Rev. B 94, 13 (2016). https://doi.org/10. 1103/PhysRevB.94.134309

22. N. E. Hussey, K. Takenaga, H. Takagi, Phil. Mag. 84, (2004). https://doi.org/10.1080/14786430410001716944. arXiv:cond-mat/0404263 [cond-mat.str-el]

23. L.V. Delacrétaz, B. Goutéraux, S .A. Hartnoll, A. Karlsson, SciPost Phys. 3(3), 025 (2017). https://doi.org/10.21468/SciPostPhys.3.3. 025. arXiv:1612.04381 [cond-mat.str-el]

24. L.V. Delacrétaz, B. Goutéraux, S .A. Hartnoll, A. Karlsson, Phys. Rev. B 96(19), 195128 (2017). https://doi.org/10.1103/PhysRevB. 96.195128. arXiv:1702.05104 [cond-mat.str-el]

25. J .M. Tranquada, J .D. Axe, N. Ichikawa, Y. Nakamura, S. Uchida, B. Nachumi, Phys. Rev. B 54, 10 (1996). https://doi.org/10.1103/ PhysRevB.54.7489

26. Y .Y. Peng, R. Fumagalli, Y. Ding, M. Minola, S. Caprara, D. Betto, M. Bluschke, G .M. De Luca, K. Kummer, E. Lefrançois, M. Saluzzo, H. Suzuki, M. Le Tacon, X .J. Zhou, N .B. Brookes, B. Keimer, L. Braicovich, M. Grilli, G. Ghiringhelli, Nat Mater 17, 697-702 (2018). https://doi.org/10.1038/s41563-018-0108-3

27. R. Arpaia, S. Caprara, R. Fumagalli, G. De Vecchi, Y. Y. Peng, E. Andersson, D. Betto, G. M. De Luca, N. Brookes, F. Lombardi, M. Saluzzo, L. Braicovich, C. Di Castro, M. Grilli, G. Ghiringhelli. arXiv: 1809.04949 [cond-mat.supr-con]

28. A. Amoretti, D. Areán, R. Argurio, D. Musso, L .A. Pando Zayas, JHEP 1705, 051 (2017). https://doi.org/10.1007/ JHEP05(2017)051. arXiv:1611.09344 [hep-th]

29. A. Amoretti, D. Areán, B. Goutéraux, D. Musso, Phys. Rev. D 97(8), 086017 (2018). https://doi.org/10.1103/PhysRevD.97. 086017. arXiv:1711.06610 [hep-th]
30. A. Amoretti, D. Areán, B. Goutéraux, D. Musso, Phys. Rev. Lett. 120(17), 171603 (2018). https://doi.org/10.1103/PhysRevLett. 120.171603. arXiv:1712.07994 [hep-th]

31. S. R. Coleman, Nucl. Phys. B 262 (1985) 263 Erratum: [Nucl. Phys. B 269 (1986) 744]. https://doi.org/ 10.1016/0550-3213(85)90286-X, https://doi.org/10.1016/ 0550-3213(86)90520-1

32. I. Amado, D. Arean, A. Jimenez-Alba, K. Landsteiner, L. Melgar, I.S. Landea, Holographic Type II Goldstone bosons. JHEP 1307, 108 (2013). https://doi.org/10.1007/JHEP07(2013)108. arXiv: 1302.5641 [hep-th]

33. A. Donos, D. Martin, C. Pantelidou, V. Ziogas, Hydrodynamics of broken global symmetries in the bulk. JHEP 1910, 218 (2019). https://doi.org/10.1007/JHEP10(2019)218. arXiv:1905.00398 [hep-th]

34. A. Amoretti, D. Areán, B. Goutéraux and D. Musso, "Gapless and gapped holographic phonons," arXiv:1910.11330 [hep-th]

35. M. Ostrogradsky, Mem. Acad. St. Petersbourg 6(4), 385 (1850)

36. R .P. Woodard, Scholarpedia 10(8), 32243 (2015). https://doi.org/ 10.4249/scholarpedia.32243. arXiv:1506.02210 [hep-th]

37. M. Nitta, S. Sasaki, R. Yokokura,. arXiv:1706.02938 [hep-th]

38. E. Rabinovici, Lect. Notes Phys. 737, 573 (2008)

39. E. Rabinovici, Les Houches 87, 217 (2008). arXiv:0708.1952 [hepth]

40. N. Manton, P. Sutcliffe, Cambridge University Press (2004)

41. R. Argurio, G. Giribet, A. Marzolla, D. Naegels, J.A. Sierra-Garcia, JHEP 1704, 007 (2017). https://doi.org/10.1007/ JHEP04(2017)007. arXiv:1612.00771 [hep-th]

42. S k Ma, R. Rajaraman, Phys. Rev. D 11, 1701 (1975). https://doi. org/10.1103/PhysRevD.11.1701

43. N.I. Gushterov, R. Rodgers, R. Rodgers,. arXiv:1807.11327 [hepth]

44. A. Amoretti, D. Areán, B. Goutéraux, D. Musso, arXiv: 1812.08118 [hep-th]

45. R. Argurio, A. Marzolla, A. Mezzalira, D. Musso, JHEP 1603, 012 (2016). https://doi.org/10.1007/JHEP03(2016)012. arXiv:1512.03750 [hep-th]

46. R. Argurio, A. Marzolla, A. Mezzalira and D. Naegels, Phys. Rev. D 92 (2015) no.6, 066009 10.1103/PhysRevD.92.066009 arXiv:1507.00211 [hep-th]

47. R. Argurio, J. Hartong, A. Marzolla, D. Naegels, JHEP 1802, 053 (2018). https://doi.org/10.1007/JHEP02(2018)053. arXiv: 1709.08383 [hep-th]

48. L.D. Landau, Zh Eksp, Teor. Fiz. 7, 19 (1937)

49. L.D. Landau, Phys. Z. Sowjetunion 11, 26 (1937)

50. L.D. Landau, Ukr. J. Phys. 53, 25 (2008)

51. H.B. Nielsen, S. Chadha, Nucl. Phys. B 105, 445 (1976). https:// doi.org/10.1016/0550-3213(76)90025-0

52. H. Watanabe, T. Brauner, Phys. Rev. D 84, 125013 (2011). https:// doi.org/10.1103/PhysRevD.84.125013. arXiv:1109.6327 [hep-ph]

53. H. Watanabe, H. Murayama, Phys. Rev. Lett. 108, 251602 (2012). https://doi.org/10.1103/PhysRevLett.108.251602. arXiv:1203.0609 [hep-th]

54. A. Kapustin,. arXiv:1207.0457 [hep-ph]

55. A. Nicolis, R. Penco, R .A. Rosen, Phys. Rev. D 89(4), 045002 (2014). https://doi.org/10.1103/PhysRevD.89.045002. arXiv:1307.0517 [hep-th]

56. O. Luongo, H. Quevedo, Int. J. Mod. Phys. D 23, 1450012 (2014). https://doi.org/10.1142/S0218271814500126

57. P. Creminelli, G. D'Amico, J. Norena, L. Senatore, F. Vernizzi, JCAP 1003, 027 (2010). https://doi.org/10.1088/1475-7516/2010/ 03/027. arXiv:0911.2701 [astro-ph.CO]

58. R. Elliott, R. Lange, Phys. Rev. 152 (1966) 1, 235-239 https://doi. org/10.1103/PhysRev.152.235

59. T. Brauner, Symmetry 2, 609 (2010). https://doi.org/10.3390/ sym2020609. arXiv:1001.5212 [hep-th] 
60. T. Brauner, S. Moroz, arXiv:1806.10441 [cond-mat.supr-con]

61. A. Biasi, P. Carracedo, J. Mas, D. Musso, A. Serantes, JHEP 1804, 137 (2018). https://doi.org/10.1007/JHEP04(2018)137. arXiv:1712.07637 [hep-th]

62. P. Carracedo, J. Mas, D. Musso, A. Serantes, JHEP 1705, 141 (2017). https://doi.org/10.1007/JHEP05(2017)141. arXiv:1612.07701 [hep-th]

63. J. Polchinski, In *Boulder 1992, Proceedings, Recent directions in particle theory* 235-274, and Calif. Univ. Santa Barbara - NSF-
ITP-92-132 (92,rec.Nov.) 39 p. (220633) Texas Univ. Austin UTTG-92-20 (92,rec.Nov.) 39 p [hep-th/9210046]

64. D. Bolmatov, E. T. Musaev, K. Trachenko, Nat. Sci. Rep. 32794 https://doi.org/10.1038/srep02794

65. D. Bolmatov, D. Zav'yalov, M. Zhernenkov, E .T. Musaev, Y .Q. Cai, Ann. Phys. 363, 221-242 (2015). https://doi.org/10.1016/j. aop.2015.09.018 